\title{
Applying branching processes to delay-tolerant networks
}

\author{
Dieter Fiems ${ }^{1}$ and Eitan Altman ${ }^{2}$ \\ 1 SMACS Research Group, Department TELIN, Ghent University, Belgium \\ 2 INRIA, BP93, 06902 Sophia Antipolis, France
}

\begin{abstract}
Mobility models that have been used in the past to study delay tolerant networks (DTNs) have been either too complex to allow for deriving analytical expressions for performance measures, or have been too simplistic. In this paper we identify several classes of DTNs where the dynamics of the number of nodes that have a copy of some packet can be modelled as branching process with migration. Using recent results on such processes in a random environment, we obtain explicit formulae for the first two moments of the number of copies of a file that is propagated in the DTN, for quite general mobility models. Numerical examples illustrate our approach.
\end{abstract}

\section{Introduction}

Delay tolerant networks (DTNs) embrace the concept of occasionally-connected networks $[7,10]$, such as sensor networks, wireless networks with alternating connectivity, etc. In this paper, we address packet forwarding in DTNs where connectivity is low and nodes relay packets of other nodes. We focus on two-hop routing schemes [15] in which a relay node that receives a packet from the source does not relay it further to other intermediate nodes. (Such a restriction may be needed in the context of resource limitations or for security reasons.) We show that various dynamics of packet forwarding in DTNs can be described by multitype branching processes with immigration operating in a random environment. We then use novel tools from branching processes with immigration in order to derive the two first moments of the number of nodes with a copy of the file.

Related work. Before proceeding to the main results, we present a brief overview of the scientific context of the branching processes methodology and to their applications in networking. The first results on branching processes are often attributed to Galton and Watson and date back to the 19th century. At that time, there was a severe concern among aristocratic families that the surnames were becoming extinct. The disappearance of a name of a family was considered as the death of the family and it was thought that the extinct families were replaced by families from lower social layers [2]. F. Galton posed the question of computing the extinction probability of the names in the Educational Times of 1873 [13]. More precisely, assume that each man in generation $n$ has some 
random number of sons in generation $n+1$, according to a fixed probability distribution that does not vary from individual to individual. What is then the probability that a family dies out? The Reverend Henry William Watson replied with a solution [26]. Together, they then wrote an 1874 paper entitled "On the probability of extinction of families" [14]. Galton and Watson appear to have derived their process independently of the much earlier work by the French statistician I. J. Bienaymé [5] (1845), which was unknown till it was rediscovered in 1962 by Heyde and Senneta, see e.g. [17].

Branching processes with a random environment have been well studied, both with and without immigration, see [4]. For example, conditions are presented for the extinction when the random environment is stationary ergodic. The stability, strong law of large numbers and central limit theorems for multitype branching processes with immigration in a random environment have been studied in $[18,24]$. These processes find applications in very diverse fields, including biological systems and queueing theory. For example, McNamara et. Al [21] consider an asexual species with non-overlapping generations. Individuals born in some year, reach maturity and reproduce one year later and then die. The number of individuals of the different genotypes in the consecutive years constitute a multi-type branching process. Prime examples in queueing theory where branching processes with immigration play a major role, include infinite server queues [9], processor sharing queues [16,22], as well as various polling systems $[3,23]$. The infinite server queue with random environment has been studied recently in $[8,11]$. These authors assume a independent exponentially distributed interarrival and service times. The theoretical framework applied here allows for explicit expressions for the first and second moments in the more general setting of general stationary ergodic processes describing the contact processes between pairs of nodes and general independent bounded service time, with a Markovian random environment. It builds on the Theory we developed in [12] and in references therein that allows to compute explicitly the two first moments of the branching process for the case of general stationary ergodic immigration process.

\section{Theoretical framework}

First, we briefly present the standard (basic) scalar branching process taking integer values. We then present several extensions, including the vector (multitype) case. In particular, we introduce the framework of [12] that extends branching processes, and yet provides explicit expressions for the first two moments.

\subsection{The scalar integer-valued case}

The standard branching is defined as follows. Let $X_{n}$ be the number of individuals in generation $n$. Starting with a fixed $X_{0}$, we define recursively

$$
X_{n+1}=\sum_{i=1}^{X_{n}} \xi_{n}^{(i)}
$$


where $\xi_{n}^{(i)}$ are independent and identically distributed random variables taking non-negative integer values. Define $A_{n}(m):=\sum_{i=1}^{m} \xi_{n}^{(i)}$ we can rewrite the above as

$$
X_{n+1}=A_{n}\left(X_{n}\right) .
$$

Branching processes with immigration are defined through the recursion

$$
X_{n+1}=A_{n}\left(X_{n}\right)+B_{n} .
$$

From equations (1) and (2), $A_{n}$ obviously possess a divisibility property; for any non-negative integers $m, m_{1}$ and $m_{2}$ such that $m_{1}+m_{2}=m$, and for any $n$,

$$
A_{n}(m)=A_{n}^{(1)}\left(m_{1}\right)+A_{n}^{(2)}\left(m_{2}\right)
$$

where for each $n, A_{n}^{(1)}$ and $A_{n}^{(2)}$ are independent random processes, both with the same distribution as $A_{n}$.

This divisibility property naturally leads to the definition of branching processes on a continuous state space. We take this property, together with the nonnegativity of $A_{n}$ as the basis to define the continuous state branching processes. Noting that these properties are satisfied by Lévy processes, we define a continuous state branching process as one satisfying (2) where $A_{n}$ is a non-negative Lévy process. For references as well as for alternative (equivalent) definitions, see $[1,6,19,20]$ and the references therein.

\subsection{General setting}

Consider the sequence of random column vectors $X_{n} \in \mathbb{R}^{M}$, adhering to,

$$
X_{n+1}=A_{n}\left(X_{n}, Y_{n}\right)+B_{n}\left(Y_{n}\right), \quad n \in \mathbb{Z},
$$

The process $Y_{n}$ and the vector valued processes $A_{n}$ and $B_{n}$ correspond to the environment process, the branching process and the immigration process, respectively. The random environment $Y_{n}$ is a stationary ergodic Markov chain, taking values on a finite state-space $\Theta=\{1,2, \ldots, N\}$; let $P=\left[p_{i j}\right]$ denote its transition matrix. The branching processes $A_{n}: \mathbb{R}^{M} \times \Theta \rightarrow \mathbb{R}^{M}$ are independent and identically distributed and further adhere to the following assumptions.

- For each $i \in \Theta, A_{n}(\cdot, i)$ has a divisibility property. Let $x=x^{1}+x^{2}+\ldots+x^{k} \in$ $\mathbb{R}^{M}$, then $A_{n}(x, i)$ has the following representation,

$$
A_{n}(x, i)=\sum_{l=1}^{k} \hat{A}_{n}^{(l)}\left(x^{l}, i\right),
$$

whereby $\hat{A}_{n}^{(l)}(\cdot, i), l=1, \ldots, k$, are identically distributed, but not necessarily independent, with the same distribution as $A_{n}(\cdot, i)$. Branching processes are those in which $\hat{A}_{n}^{(l)}(\cdot, i), l=1, \ldots, k$, are independent. 
- For each $i \in \Theta$ and $x=\left[x_{1}, \ldots, x_{M}\right] \in \mathbb{R}^{M}$, the first and second order moments of $A_{n}(\cdot, i)$ can be expressed as follows,

$$
\mathrm{E}\left[A_{n}(x, i)\right]=\mathcal{A}_{i} x, \quad \mathrm{E}\left[A_{n}(x, i) A_{n}^{\prime}(x, i)\right]=F_{i}\left(x x^{\prime}\right)+\sum_{j=1}^{M} x_{j} \Gamma_{i, j},
$$

whereby $\mathcal{A}_{i}$ and $\Gamma_{i, j}$ are fixed $M \times M$ matrices and $F_{i}$ is a linear operator that maps $M \times M$ non-negative definite matrices on $M \times M$ non-negative definite matrices and satisfies $F_{i}(0)=0$.

Finally, the immigration process $B_{n}: \Theta \rightarrow \mathbb{R}^{M}$ is a stationary ergodic sequence of random functions. The first and second order moments are denoted by $b_{i}=$ $\mathrm{E}\left[B_{0}(i)\right]$ and $\mathcal{B}_{i j}^{(n)}=\mathrm{E}\left[B_{0}(i) B_{n}(j)\right]$.

Before proceeding to the main theorems, some notation is introduced. Let $\hat{\mathcal{A}}$ denote the block matrix whose $i j$ th block entry is given by $\mathcal{A}_{j} p_{j i}(i, j \in \Theta)$. Moreover, the following block vector and block matrix simplify notation,

$$
\hat{b}=\sum_{i \in \Theta} \pi_{i}\left[\begin{array}{c}
p_{i 1} b_{i} \\
p_{i 2} b_{i} \\
\vdots \\
p_{i N} b_{i}
\end{array}\right], \hat{\mathcal{B}}^{(n)}=\sum_{i \in \Theta} \pi_{i}\left[\begin{array}{cccc}
\mathcal{B}_{i 1}^{(n)} p_{i 1} & \mathcal{B}_{i 2}^{(n)} p_{i 1} & \ldots & \mathcal{B}_{i N}^{(n)} p_{i 1} \\
\mathcal{B}_{i 1}^{(n)} p_{i 2} & \mathcal{B}_{i 2}^{(n)} p_{i 2} & \ldots & \mathcal{B}_{i N}^{(n)} p_{i 2} \\
\vdots & & \ddots & \\
\mathcal{B}_{i 1}^{(n)} p_{i N} & \mathcal{B}_{i 2}^{(n)} p_{i N} & \ldots & \mathcal{B}_{i N}^{(n)} p_{i N}
\end{array}\right]
$$

The existence of a stationary solution is now asserted by the following theorem. Theorem 2 then provides expressions for the first and second order moments of this solution. The proofs of these theorems can be found in [12].

Theorem 1. Assume that (i) $b_{i}<\infty$ component-wise for all $i \in \Theta$; and (ii) that all the eigenvalues of the matrix $\hat{\mathcal{A}}$ are within the open unit disk. Then, there exist a unique stationary solution $X_{n}^{*}$, for $n \in \mathbb{Z}$ such that $\lim _{n \rightarrow \infty}\left\|X_{n}-X_{n}^{*}\right\|$ $=0$, almost surely, for any initial value $X_{0}$.

Theorem 2. Assume that the conditions of Theorem 1 are satisfied. The conditional first moment vector is then given by,

$$
\mu=\left[\mathrm{E}\left[X_{0}^{*} 1\left\{Y_{0}=i\right\}\right]\right]_{i \in \Theta}=(\mathcal{I}-\hat{\mathcal{A}})^{-1} \hat{b} .
$$

Under the additional assumption that the second order moments of $B_{0}(i)$ are finite, $i \in \Theta$, the elements $\Omega_{i}$ of the conditional second moment matrix of $X_{0}^{*}$ are the unique solution of the system of equations,

$$
\begin{aligned}
\Omega_{l} & =\mathrm{E}\left[X_{0}^{*}\left(X_{0}^{*}\right)^{\prime} 1\left\{Y_{0}=l\right\}\right] \\
& =\sum_{k \in \Theta}\left(F_{k}\left(\Omega_{k}\right)+\sum_{j=1}^{M} \mu_{k}^{(j)} \Gamma_{k}^{(j)}+\mathcal{B}_{k k}^{(0)} \pi_{k}+\mathcal{A}_{k} \Lambda_{k}+\Lambda_{k}^{\prime} \mathcal{A}_{k}^{\prime}\right) p_{k l},
\end{aligned}
$$

$l \in \Theta$, where $\Lambda_{k}$ denotes the $k$ th diagonal (block) element of $\sum_{j=0}^{\infty} \hat{\mathcal{A}}^{j} \hat{\mathcal{B}}^{(j+1)}$ and with $\mu_{k}^{(j)}$ the $j$ th element of $\mu_{k}=\mathrm{E}\left[X_{0}^{*} 1\left\{Y_{0}=k\right\}\right]$. 


\section{$3 \quad$ DTNs with packet discarding}

Consider a network with $N$ mobile nodes. A fixed node wishes to send a packet to a destination node. As connectivity is assumed to be low, the source makes use of the mobility of other nodes that serve as relays. Whenever the source is within the transmission range of another node, it transmits a packet to that node. The packet reaches its destination whenever a node with a copy of the packet is within the transmission range of the destination node. In this example as well as in all the following ones, a two-hop routing scheme is adopted [15]. A relay node that receives a packet from the source does not relay it further to other intermediate nodes. Time is discrete and at each time $n$, each node has a probability $p_{i} \geq p>0$ to meet the source node; this probability also depends on the state $i \in \Theta$ of a modulating Markov chain which models fluctuations in the channel conditions.

In order to avoid packets to remain forever at nodes, it has been suggested to use expiration timers [25]. A timer is initiated when a node receives the packet which is discarded when the timer expires. We assume that the timers are independent geometrically distributed random variables with mean packet discarding time $T$.

Let $X_{n}$ denote the number of nodes with the packet at time $n$ (excluding the source node). We have the following recursion,

$$
X_{n+1}=\sum_{i=1}^{X_{n}} \zeta_{n}^{(i)}+\sum_{i=1}^{N-X_{n}} \xi_{n}^{(i)} .
$$

Here, $\xi_{n}^{(i)}$ and $\zeta_{n}^{(i)}$ are indicators that equal 1 if the $i$ th node without the packet at time $n$ receives the packet at time $n+1$ and if the $i$ th copy of the packet is not discarded at time $n$, respectively. The recursion is not directly covered by our theoretical framework. However, by introducing a dummy variable $Y_{n}$, we may rewrite the equation as follows,

$$
\left[\begin{array}{c}
X_{n+1} \\
Y_{n+1}
\end{array}\right]=A_{n}\left(\left[\begin{array}{c}
X_{n} \\
Y_{n}
\end{array}\right]\right)+B_{n}, A_{n}\left(\left[\begin{array}{l}
x \\
y
\end{array}\right]\right)=\left(\sum_{i=1}^{x} \zeta_{n}^{(i)}+\sum_{i=1}^{y} \xi_{n}^{(i)}\right)\left[\begin{array}{c}
1 \\
-1
\end{array}\right], B_{n}=\left[\begin{array}{c}
0 \\
N
\end{array}\right]
$$

which shows that our framework is applicable.

Figure 1 depicts the mean and variance of the number of nodes that have the packet vs. $T$. The Markovian environment has two states, state 1 (2) corresponding to bad (good) channel conditions. The following transition probabilities are chosen: $\left[p_{11}, p_{22}\right]=[17 / 18,1 / 2]$ for set $1,[89 / 90,9 / 10]$ for set 2 and $[449 / 450,49 / 50]$ for set 3 . The fraction of time the chain is in state 1 is equal for all three sets. However, in comparison with set 1 , the chain stays in state 1 on average 5 times (25 times) longer for set 2 (set 3 ). Moreover, we have $N=200$ nodes and transmission probabilities $p_{1}=0.5 \%$ and $p_{2}=10 \%$. Packets remain longer with a node as $T$ increases such that the mean number of nodes that have the packet increases as well. In contrast, the variance first increases and then decreases again. Moreover, correlation in the random environment negatively affects the mean number of nodes with the packet while the variance increases. 

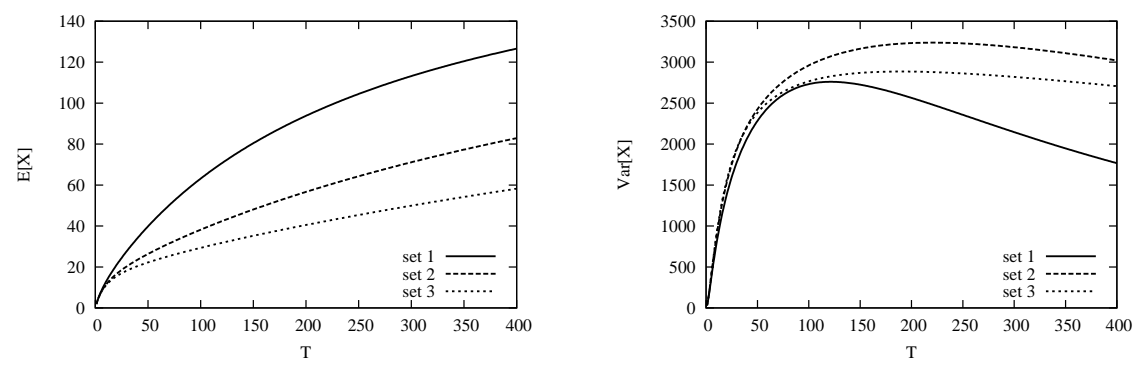

Fig. 1. Mean (left) and variance (right) of the number of nodes that have the packet as a function of the mean discarding time for various parameter settings.

\section{Variations in the total number of mobiles}

We retain the model of the previous section but now allow for variations in the number of mobile nodes and no longer include expiration timers. New nodes arrive and nodes depart from the system. Let $W_{n}$ denote the number of nodes that have the packet at slot $n$ and let $Z_{n}$ denote the number of nodes that do not have the packet. We have the following recursion,

$$
W_{n+1}=\sum_{j=1}^{W_{n}} \zeta_{n, 1}^{(j)}+\sum_{j=1}^{Z_{n}} \zeta_{n, 2}^{(j)} \nu_{n}^{(j)}, Z_{n+1}=\sum_{j=1}^{Z_{n}} \zeta_{n, 2}^{(j)}\left(1-\nu_{n}^{(j)}\right)+B_{n} .
$$

Here $\zeta_{n, 1}^{(j)}$ is the indicator that the $j$ th node that has the packet leaves the system at slot $n, \zeta_{n, 2}^{(j)}$ is the indicator that the $j$ th node that does not have the packet leaves the system at slot $n$ and $\nu_{n}^{(j)}$ is the indicator that the $j$ th node that does not have the packet, receives the packet at slot $n$. Finally, $B_{n}$ denotes the number of new nodes that arrive during slot $n$. Assuming stationary ergodic arrivals of nodes and independent geometrically distributed residence times with mean $T$, the theoretical framework applies.

Still assuming a Markovian environment with two states, its transition probabilities are characterised by the fraction $\sigma$ that the environment is in state 1 and by the mean time $\tau$ to alternate from state 1 to state 2 and back. Figure 2 depicts the mean number of nodes $\mathrm{E}[W]$ that have the packet and the mean number of nodes $\mathrm{E}[Z]$ that do not have the packet. The left pane plots these means vs. $T$ for different values of $\tau$. The mean number of nodes in the system is fixed to 50 by scaling the mean number of arrivals $\mathrm{E}[B]$ in a slot for increasing $T$. In state 2 , a node receives the packet with probability $p_{2}=0.1$ whereas no transmission is possible in state $1\left(p_{1}=0\right)$. Moreover, for all curves, $\sigma=90 \%$. It is readily observed that the mean residence time of a node has a considerable impact on $\mathrm{E}[W]$. Obviously, if nodes remain longer, they carry the packet for a longer time which explains the increase in the mean number of nodes that carry the packet. Further, increasing $\tau$ yields lower values of $\mathrm{E}[W]$. This is confirmed 

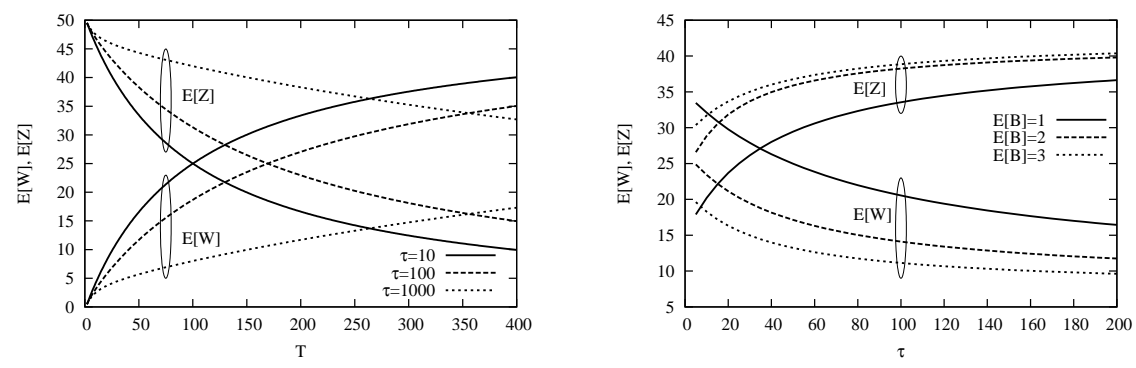

Fig. 2. Mean number of nodes that have the packet and of the number of nodes that do not have the packet vs. $T$ for various values of $\tau$ (left) and vs. $\tau$ for various values of $\mathrm{E}[B]$ (right).

by the right pane where $\mathrm{E}[Z]$ and $\mathrm{E}[W]$ are depicted vs. $\tau$ for various values of $\mathrm{E}[B]$ and the same parameter settings.

\section{Mobility of the source and the nodes}

We retain the model of the previous section but now replace the channel model by a mobility model. The source node moves according to a random walk through the spatial grid, depicted in Figure 3 (left). In each of the regions of the grid, new nodes arrive according to a stationary ergodic process which then travel through the grid until they leave. If a node is in the same region as the source, the node receives the packet with a fixed (possibly region-dependent) probability.

Let $X_{n}(k)$ denote the number of nodes in region $k$ at time $n$ with the packet and let $Z_{n}(k)$ denote the number of nodes without the packet. Further let $X_{n}$ and $Z_{n}$ denote the column vectors with elements $X_{n}(k)$ and $Z_{n}(k)$, respectively. Let $Y_{n}$ denote the region where the source node resides at time $n$ - the environment thus tracks the position of the source node - and let $B_{n}(k)$ denote the number of new nodes that arrive in region $k$ at time $n ; B_{n}$ is a column vector with elements $B_{n}(k)$. We then have the following recursion,

$X_{n+1}=\sum_{i=1}^{N} \sum_{j=1}^{X_{n}(i)} \zeta_{n, 1}^{(i, j)}+\sum_{i=1}^{N} \sum_{j=1}^{Z_{n}(i)} \zeta_{n, 2}^{(i, j)} \nu_{n}^{(i, j)}, Z_{n+1}=\sum_{i=1}^{N} \sum_{j=1}^{Z_{n}(i)} \zeta_{n, 2}^{(i, j)}\left(1-\nu_{n}^{(i, j)}\right)+B_{n}$.

Here $\zeta_{n, 1}^{(i, j)}$ is a column vector of indicators; its $k$ th element is the indicator that the $j$ th node in region $i$ that has the packet at time $n$ moves to region $k$. The indicator vector $\zeta_{n, 2}^{(i, j)}$ is defined likewise. Its $k$ th element is the indicator that the $j$ th node in region $i$ that does not have the packet at time $n$ moves to region $k$. Further, $\nu_{n}^{(i, j)}$ denotes the indicator that the $j$ th node in region $i$ that does not have the packet at time $n$, receives the packet. Notice that some of the packets may leave the grid as not all packets necessarily move to any of the regions. Assuming geometrically distributed residence times (possibly region dependent) and random routing between the regions, the theoretical framework is applicable. 

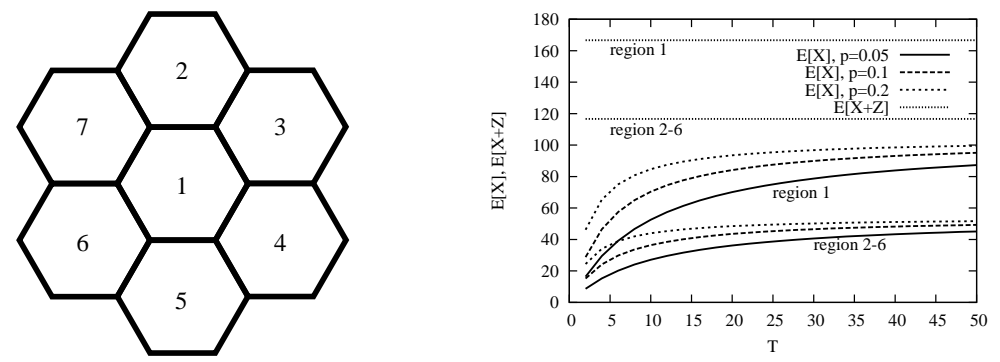

Fig. 3. Spatial grid of the nodes (left) and mean number of nodes with the packet and mean number of nodes vs. the mean residence time in a region (right)

To limit the number of parameters involved, we assume that the mean residence times in the different regions are equal and nodes move to any of the neighbouring regions with probability $1 / 6$, thereby possibly leaving the grid (however, the source node never leaves the grid). A node that does not have the packet which is in the same region as the source node, receives the packet with probability $p$. The right pane of Figure 3 depicts the mean number of nodes with the packet in the different regions is vs. $T$ for different values of the transmission probability $p$. The mean numbers of new arrivals in the different regions scale with the residence times of the nodes: $\mathrm{E} B^{(i)}=50 / T$ for $i=1,2, \ldots, 7 \mathrm{such}$ that the total mean number of nodes in the different regions $\mathrm{E}[X+Z]$ remains constant. First, notice that by symmetry, the characteristics of regions 2 to 7 are the same. Further, it is clear that longer residence times imply that more nodes receive the packet. Clearly, nodes do not only remain longer in a region but also longer in the grid. Hence, the probability that they receive the packet increases.

\section{Conclusions}

In this paper, it was shown that some of the dynamics of packet forwarding in DTNs can be described by Markov-modulated branching processes with immigration. Three models for two-hop packet forwarding were developed: the number of nodes was either fixed, variable, or variable and mobile.

\section{References}

1. S. R. Adke and V. G. Gadag, "A new class of branching processes", Branching Processes: Proceedings of the First World Congress, C.C.Heyde (Editor), 1-13, Springer Lecture Notes 99, 1995.

2. K. Albertsen, The Extinction of Families, International Statistical Review / Revue Internationale de Statistique, Vol. 63, No. 2 (Aug., 1995), pp. 234-239.

3. E. Altman and D. Fiems. Expected waiting time in polling systems with correlated vacations. Queueing Systems, 56(3-4):241-253, 2007. 
4. K. Athreya and A. Vidyashankar. Handbook of Statistics, volume 19, chapter Branching Processes. Elsevier Science B.V., 2001.

5. I. J. Bienaymé, "De la loi de la multiplication et de la durée des familles", Soc. Philomath., Paris Extraits Ser. 5, 37-39, 1845.

6. J. Bertoin. Lévy Processes. Cambridge University Press, 2002.

7. V. Cerf et Al. Delay-tolerant network architecture. IETF RFC 4838.

8. B. D'Auria. $\mathrm{M} / \mathrm{M} / \infty$ queues in semi-markovian random environment. Queueing Systems, 58(3):221-237, 2008.

9. I. Eliazar. On the discrete-time $G / G I / \infty$ queue. Technical report, Holon Institute of Technology, 2006.

10. K. Fall. A delay-tolerant network architecture for challenged internets. In Proceedings of SIGCOMM 2003, pp. 27-34, 2003.

11. G. Falin. The $\mathrm{M} / \mathrm{M} / \infty$ queue in a random environment. Queueing Systems, $58(1): 65-76,2008$.

12. D. Fiems and E. Altman. Markov-modulated stochastic recursive equations with applications to delay-tolerant networks. INRIA Research Report No. 6872, 2009. Submitted to Performance Evaluation.

13. F. Galton, Problem 4001. Educational Times April 1 (17), 1873.

14. F. Galton and H. W. Watson, "On the probability of the extinction of the families", J. Royal Antropol. Soc., London, vol. 4, pp. 138-144, 1874.

15. M. Garetto, P. Giaccone, and E. Leonardi. On the effectiveness of the 2-hop routing strategy in mobile ad hoc networks. In Proceedings of ICC '07, 2007.

16. S. A. Grishechkin. On a relation between processor sharing queues and CrumpMode-Jagers branching processes. Advances in Applied Probability, 24:653-698, 1992.

17. C. C. Heyde and E. Senneta, Studies in the History of Probability and Statistics. XXXI. The simple branching process, a turning point test and a fundamental inequality: A historical note on I. J. Bienaymé, Biometrika 1972 59(3):680-683, 1972.

18. E. Key. Limiting distributions and regeneration times for multitype branching processes with immigration in a random environment. Annals of Probability, 15:344353, 1987.

19. A. Lambert. The genealogy of continuous-state branching processes with immigration. Journal of Probability Theory and Related Fields, 122(1):42-70, 2002.

20. J. F. Le Gall, Random trees and spatial branching processes, Maphysto Lecture Notes Series (Univ of Aarhus), vol 9, 2000.

21. J. McNamara, A. Houston, and E. Collins. Optimality models in behavioral biology. SIAM Review, 43(3):413-466, 2001.

22. R. Núñez Queija. Processor-Sharing Models for Integrated-Services Networks. PhD thesis, Eindhoven University of Technology, 2000.

23. J. Resing. Polling systems and multi-type branching processes. Queueing Systems, 13:409-426, 1993.

24. A. Roitershtein. A note on multitype branching processes with immigration in a random environment. Annals of Probability, 35(4):1573-1592, 2007.

25. T. Small and Z. Haas. Resource and performance tradeoffs in delay-tolerant wireless networks. In Proceedings of the ACM SIGCOMM workshop on Delay Tolerant Networks, Philadelphia, August 2005.

26. H.W. Watson, Solution to problem 4001. Educational Times August 1, 115-116, 1873. 